\title{
Isolated hip and ankle fatigue are unlikely risk factors for anterior cruciate ligament injury
}

\author{
A. C. Thomas ${ }^{1}$, R. M. Palmieri-Smith ${ }^{1,2,3}$, S. G. McLean ${ }^{1,2}$ \\ ${ }^{1}$ School of Kinesiology, University of Michigan, Ann Arbor, Michigan, USA, ${ }^{2}$ Bone and Joint Injury Prevention and Rehabilitation \\ Center, University of Michigan, Ann Arbor, Michigan, USA, ${ }^{3}$ Department of Orthopaedic Surgery, University of Michigan, Ann \\ Arbor, Michigan, USA \\ Corresponding Author: Abbey Thomas, School of Kinesiology, University of Michigan, 401 Washtenaw Ave, Ann Arbor, \\ Michigan 48109-2214, USA.Tel: +1 734647 3871,Fax: +1 734936 1925,E-mail: abbeyt@umich.edu
}

Accepted for publication 30 November 2009

\begin{abstract}
Lower extremity neuromuscular fatigue purportedly increases anterior cruciate ligament (ACL) injury risk through promotion of extreme landing mechanics. However, the impact of fatigue on muscle groups critical to the landing strategy remains unclear. This study examined the effects of isolated hip rotator and triceps surae fatigue on lower extremity landing biomechanics. Sixteen healthy females (18-22 years) reported for testing on two occasions, with one muscle group fatigued per session. Subjects performed three single-leg landings onto a force platform pre- and postfatigue, defined as an $80 \%$ decrease in peak torque in the targeted muscle group. Hip rotator fatigue was induced via alternating concentric contractions and triceps surae
\end{abstract}

fatigue through concentric plantar flexion contractions on an isokinetic dynamometer. Initial contact (IC) kinematics and peak stance (PS) kinetics and kinematics were analyzed pre- and post-fatigue. Hip rotator fatigue increased IC $(P=0.05)$ and $P S(P=0.04)$ hip internal rotation angles. Triceps surae fatigue decreased IC knee flexion $(P=0.01)$ angle. Isolated hip rotator and triceps surae fatigue each produced modifications in lower limb kinematic parameters viewed as risk factors for ACL injury. These modifications, however, do not appear of sufficient magnitude to compromise ligament integrity, suggesting injury via an integrative lower extremity fatigue mechanism is more likely.
Research into the causes of non-contact anterior cruciate ligament (ACL) injury typically focuses on landing neuromechanics, as such factors are, effectively, modifiable (Hewett et al., 2006). Through this research, specific neuromechanical risk factors have emerged, with an extended landing posture (Boden et al., 2000), increased knee abduction angles (Ford et al., 2005), and loads (Besier et al., 2001) during early stance being most commonly implicated. Evolving in line with these findings are training programs intent on modifying these high risk neuromechanical profiles (Hewett et al., 1999). Despite these efforts, however, and reported early successes (Hewett et al., 1999), ACL injury rates and the associated sex disparity have not decreased (Agel et al., 2005). It seems plausible, therefore, that current prevention efforts remain unable to counter critical biomechanical factors associated with the injury mechanism.

Neuromuscular fatigue, a largely inevitable occurrence in sports, has recently been implicated within non-contact ACL injury (Chappell et al., 2005; McLean et al., 2007) with epidemiological data inferring increased risk as athletes succumb to the physiological demands of intense activity (Hawkins
\& Fuller, 1999; Bradley et al., 2002). Lower extremity fatigue promotes substantial modifications in lower limb joint biomechanical profiles consistently linked to non-contact ACL injury (Chappell et al., 2005; McLean et al., 2007; Borotikar et al., 2008). An increased understanding of the role of fatigue within the ACL injury mechanism thus seems critical to improved prevention success.

Before neuromuscular fatigue can be successfully addressed within injury prevention programs, a better understanding of the precise impacts of fatigue on joint neuromechanics is needed. Currently, fatigue effects are typically examined within a general fatigue model (Chappell et al., 2005; McLean et al., 2007; McLean \& Samorezov, 2009), affording assessment of joint mechanical responses to more game-like fatigue conditions. This approach, however, does not enable determination of how specific muscle groups may govern this response. In our own work (Thomas et al., in press), moderate $(50 \%)$, isolated quadriceps and hamstrings neuromuscular fatigue was shown to substantially alter hip and knee joint landing, possibly increasing ACL injury risk. It is unclear, however, as to how the fatiguing of isolated 


\section{Thomas et al.}

hip and ankle joint musculature may alter lower limb neuromechanics during landings. With extreme knee loads viewed to arise through abnormal neuromuscular control elsewhere in the lower extremity (Zeller et al., 2003; McLean et al., 2005), extending the focus to incorporate these muscle groups appears warranted. Hip rotator muscle fatigue, for example, may impact out of plane hip postures to the extent that extreme and potentially hazardous knee frontal plane loading results (McLean et al., 2005). Gastrocnemius fatigue may promote a more quadricepsdominant landing strategy, thereby inducing larger and potentially high-risk anterior tibial shear and resultant ACL loads (DeMorat et al., 2004).

Studying the effects of isolated lower extremity muscle fatigue during dynamic activity, essentially simulating a weaker muscle or muscle group (Gandevia et al., 2002) provides immediate insight into how those muscles contribute to the joint biomechanical profile. This is critical to developing more effective ACL injury prevention programs that may minimize adverse fatigue effects and target an otherwise ineffective muscle response. With this in mind, we sought to examine the effects of isolated hip rotator and triceps surae neuromuscular fatigue on lower limb biomechanics during a dynamic landing task. Based on previous research (Chappell et al., 2005; McLean et al., 2007; Borotikar et al., 2008), we hypothesized that (1) hip rotator fatigue would increase hip internal rotation (IR) and knee abduction angles and the external knee abduction moment and (2) triceps surae fatigue would decrease knee flexion angle, increase external knee extension moment and decrease ankle plantar flexion moment during the stance phase of landing.

\section{Materials and methods \\ Subjects}

Sixteen healthy, recreationally active female volunteers (mass $57.92 \pm 6.87 \mathrm{~kg}$, height $1.64 \pm 0.05 \mathrm{~m}$ ), aged 18-22 participated. An a priori power analysis performed utilizing previously reported knee abduction rotation data (McLean et al., 2007) revealed that 15 subjects were required to achieve $80 \%$ statistical power with an $\alpha$-level of 0.05 . Subject exclusion criteria included: (1) previous history of knee injury or surgery; (2) current knee pain; (3) current hip or ankle injury; and/or (4) history of lower extremity injury in the past 6 months. The institutional review board approved the study. Subjects provided informed consent before participation.

\section{Testing procedures}

Subjects reported for testing on two occasions, with the hip rotators (internal/external) fatigued during one session and the ipsilateral triceps surae fatigued during the other. The dominant limb, defined as the leg with which subjects would kick a soccer ball (Ford et al., 2003), was the limb utilized for both fatigue sessions. Three-dimensional (3D) hip, knee, and ankle biomechanics were recorded during three single-leg landings pre- and post-fatigue at each session. Seventy-two hours separated sessions to ensure subjects were no longer experiencing fatigue (Andersson et al., 2008), which would otherwise have confounded results. Further, fatigue order was counterbalanced before subject enrollment to ensure testing order did not impact our findings. Subjects wore spandex shorts and their own athletic shoes during testing.

For the dynamic landing task, subjects were required to jump forward off both legs over a $17 \mathrm{~cm}$ box and land with the dominant limb centered on a force platform sampling at $1200 \mathrm{~Hz}$ (AMTI 0R6-7, Watertown, Massachusetts, USA), located $1 \mathrm{~m}$ away. Immediately upon landing, they aggressively jumped laterally to their non-dominant side (Borotikar et al., 2008). Landing on the right limb, for example, necessitated a lateral jump to the left. We chose to examine a singlelimb landing task since it has been studied in detail previously (Ford et al., 2006; McLean \& Samorezov, 2009) and is considered a high-risk landing strategy for sustaining ACL injury (Olsen et al., 2004). Successful trials fell within the field of view of an eight camera high-speed $(240 \mathrm{~Hz})$ motion capture system (Vicon MX; Oxford Metrics Ltd., Oxford, UK). Subjects performed three practice landings before testing, with verbal feedback provided by one experimenter (A.C.T.), ensuring adequate familiarization with the task.

\section{Fatigue}

Before beginning the fatigue protocol, baseline peak torque of the respective muscle group was quantified (e.g., for hip rotator testing, peak torque for the internal and external rotators was assessed) via an isokinetic dynamometer (Cybex 340; Cybex International Inc., Medway, Massachusetts, USA) sampling at $100 \mathrm{~Hz}$ at a speed of $180^{\circ}$ s (Bellew \& Fenter, 2006). Peak torque was defined as the maximum value recorded over five repetitions of maximum voluntary concentric contractions (MVCC). This value was additionally used to calculate percentage strength degradation and ultimately fatigue. Two minutes of rest was provided following peak torque assessment (Bellew \& Fenter, 2006) before initiating the fatiguing exercise. Specifically, continuous MVCC repetitions were performed in the targeted muscle group until torque decreased $80 \%$ from the subject's baseline peak torque value (Ochsendorf et al., 2000) for three consecutive repetitions. At this point, subjects received $20 \mathrm{~s}$ of rest then repeated this same fatigue drill until the respective torques again dropped $80 \%$ below baseline for three consecutive repetitions. MVCC-rest sequences were repeated until fatigue was achieved, defined as the point when the first five MVCCs of any given set were performed $80 \%$ below the baseline peak torque measure. Following the fatiguing exercise, subjects immediately (within $90 \mathrm{~s}$ ) completed three additional singleleg landings on the dominant limb, with neuromechanics again recorded.

\section{Hip rotators}

Subjects sat facing the dynamometer with the test leg hip flexed to $30^{\circ}$ (Delp et al., 1999) and the knee flexed to $90^{\circ}$. The lower leg was strapped to the dynamometer arm and a stabilization strap placed across the hips. Subjects were instructed to perform alternating hip internal and external rotation MVCCs through the full, available range of motion by only rotating, but not flexing, abducting or adducting the hip. The test limb was monitored throughout the fatiguing session by one investigator (A.C.T.) to ensure compliance. 
Hip and ankle fatigue alters kinematics

Triceps surae

Subjects lay supine with the test limb knee flexed to $30^{\circ}$ (Cresswell et al., 1995) and the hip in a neutral position of abduction/adduction and rotation. The test limb foot was strapped onto the footplate attachment of the dynamometer and a stabilization strap placed over the thigh. A bolster was placed under the knee to maintain its position. Subjects performed MVCCs into plantar flexion only to avoid fatiguing the ankle dorsiflexors. Specifically, once subjects reached maximal plantar flexion, they relaxed the ankle musculature completely and the investigator passively returned the foot to the starting position (ankle positioned at $90^{\circ}$ ) before immediate initiation of the next plantar flexion MVCC.

\section{Kinematic and kinetic data collection and analysis}

Joint rotations were quantified based on the $3 \mathrm{D}$ coordinates of 19 precisely attached retro-reflective markers (Fig. 1). A video recording was initially captured with each subject standing in a neutral position (McLean et al., 2004) and a kinematic model was defined, consisting of five skeletal segments (dominant limb foot, talus, shank and thigh, and the pelvis) and 15 degrees of freedom (DOF) using Visual 3D software (Version 3.9; C-motion Inc., Rockville, Maryland, USA). The pelvis was assigned six DOF relative to the global (laboratory) coordinate system, with the hip, knee, and ankle joints each defined locally (Borotikar et al., 2008) and assigned three rotational DOF, respectively (Grood \& Suntay, 1983; Wu et al., 2002).

Following the static trial, five retro-reflective markers were removed (bilateral posterior superior iliac spine, medial femoral epicondyle, and medial and lateral malleoli). The 3D marker trajectories recorded during movement trials were processed within Visual 3D to solve for the respective joint rotations at each frame. Rotations were calculated using a Cardan rotation sequence (Cole et al., 1993) and expressed relative to each subject's neutral position (McLean et al., 2007). Synchronous ground reaction force and the above kinematic data were filtered using a zero lag, second-order Butterworth filter with a $12 \mathrm{~Hz}$ cut-off frequency (McLean et al., 2007) and submitted to standard inverse dynamics analysis within the Visual 3D software (Willson \& Davis, 2008). Segment inertial properties were defined in accordance with the work of Dempster (1955). Kinetic outputs were normalized to subject body mass and height (Willson \& Davis, 2008) and represented as external moments. Kinematic and kinetic data were time-normalized to $100 \%$ of stance for graphical purposes only, with initial contact (IC) and toe-off defined as the point when the vertical GRF first exceeded and fell below $10 \mathrm{~N}$, respectively (McLean et al., 2007; Borotikar et al., 2008).

IC and peak hip, knee, and ankle 3D joint rotations over the first $50 \%$ of stance (PS) were calculated for each trial (Borotikar et al., 2008; McLean \& Samorezov, 2009). PS joint moment data were also recorded. Subject-based angles and moments obtained over each session were averaged separately and subsequently submitted to statistical analyses.

\section{Statistical analysis}

IC kinematics and PS kinematics and kinetics were analyzed via repeated measures ANOVAs. Separate analyses were performed for hip rotator and triceps surae muscle groups. Each model had one within-subjects factor, fatigue (pre and post). Bonferroni multiple comparison procedures were used for all post hoc analyses. The $\alpha$-level was set a priori at $\leq 0.05$.

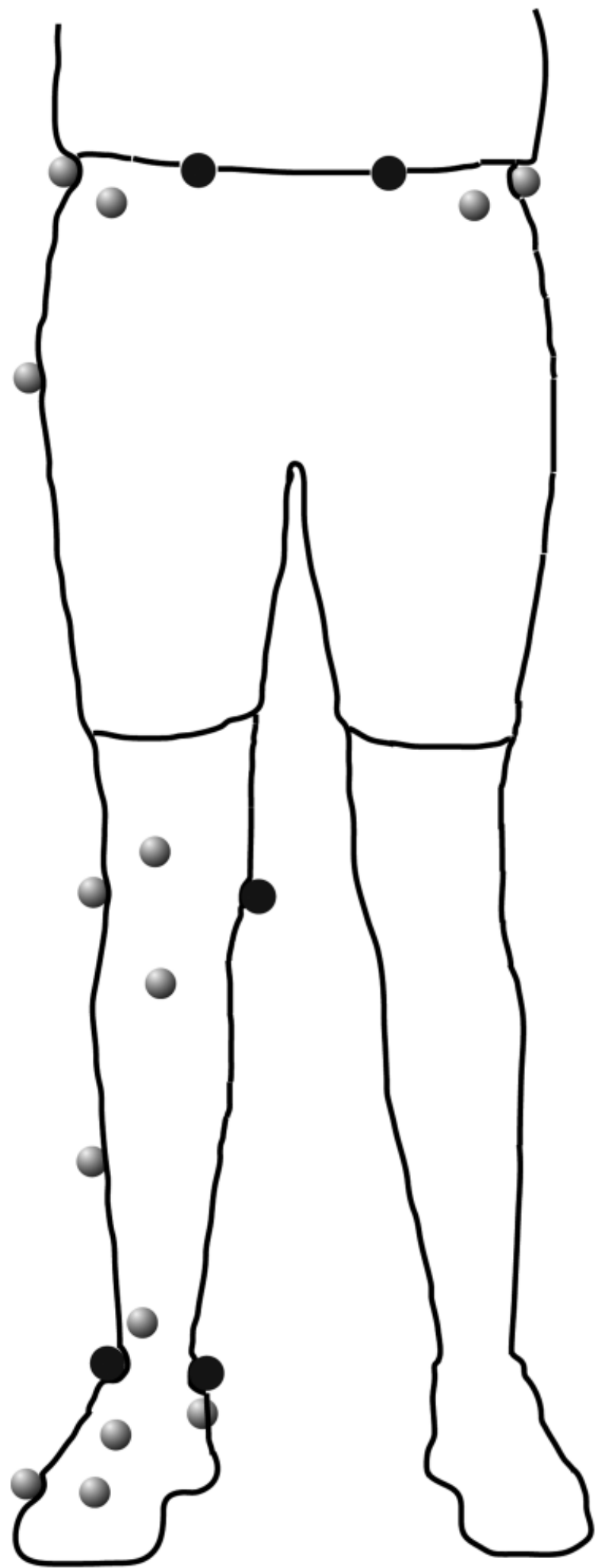

Fig. 1. Marker placements for the kinematic model. Black circles represent markers that were removed following the static trial (bilateral posterior superior iliac spine, medial femoral epicondyle, and medial and lateral malleoli). 


\section{Thomas et al.}

Effect sizes were calculated using Cohen's (1969). Statistical analyses were performed using the Statistical Package for the Social Sciences (SPSS) 16.0 software (SPSS Inc., Chicago, Illinois, USA). Effect sizes were calculated in Microsoft Excel 2007 (Microsoft Corporation, Redmond, Washington, USA).

\section{Results}

Hip rotator fatigue

A fatigue main effect was detected in the hip transverse plane following fatigue of the hip rotators $(P=0.05)$ (Table 1) (Fig. 2). Specifically, when fatigued, subjects demonstrated a significant increase in IC hip IR angle. No fatigue effects were identified for hip joint angles in the sagittal or frontal planes, for any knee joint angles, or for ankle joint angles in the sagittal or transverse planes following hip rotator fatigue $(P>0.05)$.

Hip IR angle remained increased at PS following fatigue $(P=0.04)$ (Table 2). Hip rotator fatigue did not, however, affect hip joint angles in the sagittal or frontal planes, any knee joint angles, or ankle joint angle in the sagittal or transverse planes $(P>0.05)$. Further, hip rotator fatigue did not affect kinetics at the hip, knee, or ankle $(P>0.05)$ (Table 3$)$.

\section{Triceps surae fatigue}

Following fatigue of the triceps surae musculature, a fatigue effect was identified in the knee sagittal plane $(P=0.01)$ (Table 1) (Fig. 3). Specifically in the presence of fatigue, subjects landed in a more extended posture compared with the non-fatigued state. Fatigue of the triceps surae did not, however, affect hip joint angle in any plane, knee frontal plane angles, or ankle sagittal or transverse plane angles $(P>0.05)$. Triceps surae fatigue did not alter peak hip, knee, or ankle angles (Table 2) or moments $(P>0.05)$ (Table 3).

\section{Discussion}

We sought to determine the effects of isolated hip rotator and triceps surae fatigue on lower limb landing biomechanics. Both muscle groups are crucial to lower limb control during various dynamic tasks (Decker et al., 2003); suggesting fatiguing these muscles may directly impact the movement response. Elucidating the neuromechanical adaptations arising from isolated hip rotator and triceps surae fatigue, therefore, may deepen understanding of their contributions to injurious landing patterns and afford means to better counter these adverse effects.

The fatigue model used herein fatigued either hip internal/external rotators or triceps surae muscles. We defined fatigue in each instance as a percent degradation in each subject's maximum strength (Ochsendorf et al., 2000), allowing all subjects to, theoretically, be fatigued to the same level. We acknowledge that general lower extremity fatigue models (Chappell et al., 2005; McLean et al., 2007; Borotikar et al., 2008) may better simulate fatigue effects during normal sports activity. However, investigating isolated muscle fatigue is equally important, aiding understanding of how specific muscles contribute to dynamic lower limb control and possibly ACL injury during landing tasks.

\section{Hip rotator fatigue}

As hypothesized, isolated hip rotator fatigue led to increases in hip IR angle. Subjects demonstrated hip IR throughout the entire landing phase before fatigue. With our fatiguing task directly targeting muscles responsible for controlling hip transverse plane motions, increases in hip IR postures are somewhat intuitive and potentially hazardous. Increased hip IR has been suggested to increase ACL injury risk via the promotion of extreme knee abduction motions and loads (Ireland, 2002; McLean et al., 2005). In

Table 1. Initial contact angles at the hip, knee, and ankle for pre- and post-fatigue states

\begin{tabular}{|c|c|c|c|c|c|c|c|c|}
\hline \multirow[t]{2}{*}{ Joint rotation $\left({ }^{\circ}\right)$} & \multicolumn{4}{|l|}{ Hip rotators } & \multicolumn{4}{|l|}{ Triceps surae } \\
\hline & Pre & Post & $P$ & $d$ & Pre & Post & $P$ & $d$ \\
\hline Hip flexion/extension & $36.6 \pm 6.2$ & $37.0 \pm 5.6$ & 0.73 & 0.07 & $36.8 \pm 6.6$ & $36.1 \pm 7.0$ & 0.35 & 0.10 \\
\hline Hip add/abduction & $-8.9 \pm 9.5$ & $-8.5 \pm 6.9$ & 0.61 & 0.04 & $-8.5 \pm 6.8$ & $-8.7 \pm 6.6$ & 0.73 & 0.03 \\
\hline Hip internal/external rotation & $4.7 \pm 7.0$ & $7.8 \pm 9.5$ & $0.05^{\star}$ & 0.44 & $4.3 \pm 6.8$ & $5.0 \pm 8.7$ & 0.55 & 0.10 \\
\hline Knee extension/flexion & $-15.4 \pm 4.7$ & $-13.8 \pm 5.8$ & 0.1 & 0.34 & $-14.4 \pm 5.5$ & $-12.4 \pm 5.7$ & $0.01^{*}$ & 0.36 \\
\hline Knee add/abduction & $-4.9 \pm 4.2$ & $-4.2 \pm 3.8$ & 0.14 & 0.16 & $-5.0 \pm 2.5$ & $-4.8 \pm 2.7$ & 0.38 & 0.09 \\
\hline Knee internal/external rotation & $-3.7 \pm 4.7$ & $-4.9 \pm 5.1$ & 0.11 & 0.25 & $-5.4 \pm 4.0$ & $-5.6 \pm 3.9$ & 0.67 & 0.05 \\
\hline Ankle dorsiflexion/plantar flexion & $-22.2 \pm 14.2$ & $-24.2 \pm 7.3$ & 0.47 & 0.14 & $-23.3 \pm 12.1$ & $-25.3 \pm 10.4$ & 0.41 & 0.16 \\
\hline Ankle inversion/eversion & $1.0 \pm 6.7$ & $1.8 \pm 6.9$ & 0.31 & 0.12 & $1.5 \pm 6.1$ & $2.0 \pm 6.6$ & 0.43 & 0.09 \\
\hline
\end{tabular}

All values are presented as mean \pm standard deviation.

The first rotation listed is positive (e.g., at initial contact the hip is internally rotated as indicated by the positive values).

*Statistically significant difference between fatigue conditions. 
Hip and ankle fatigue alters kinematics
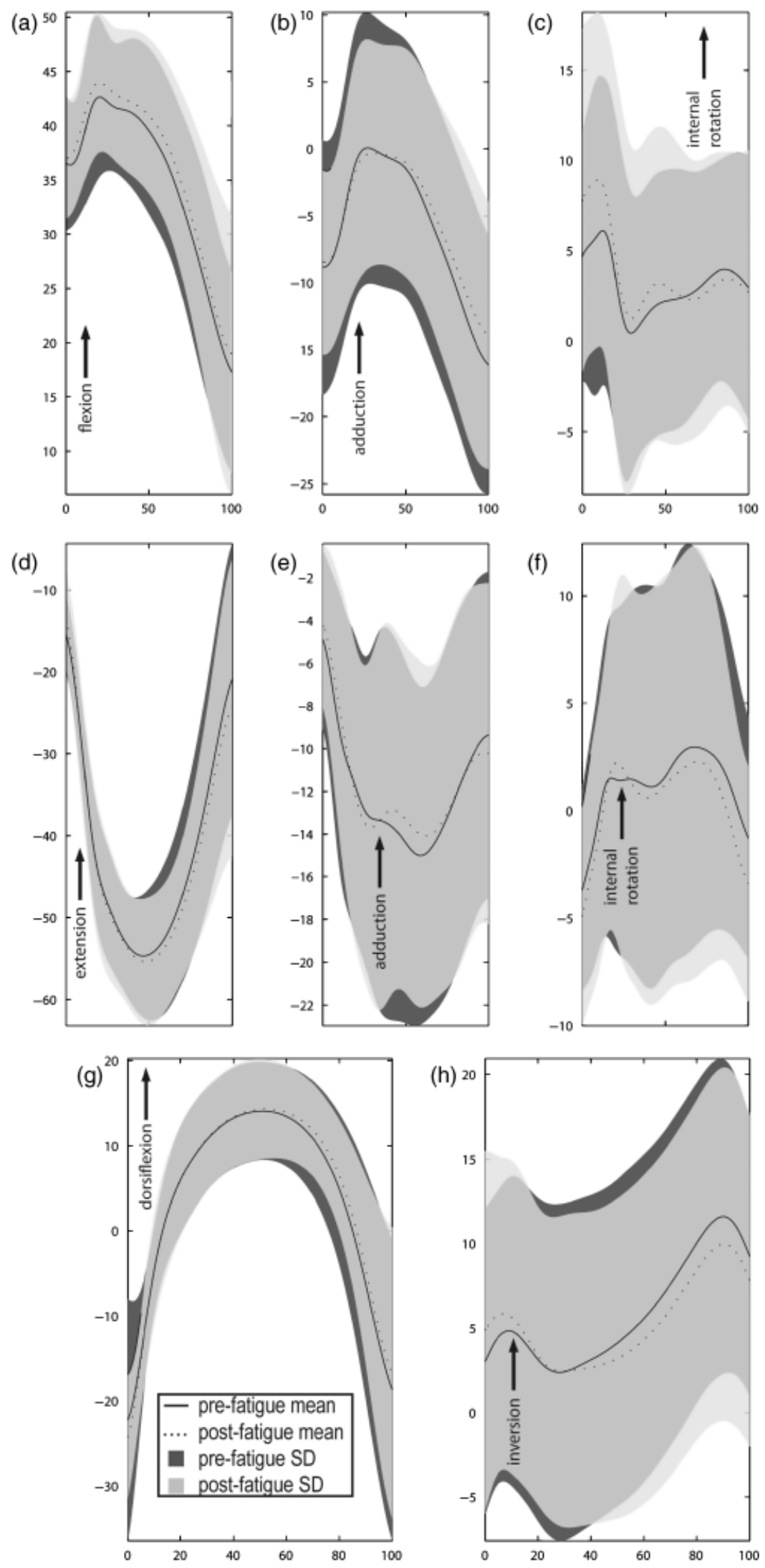

Fig. 2. Average joint rotations pre- and post-fatigue of the hip rotators. Rotations shown are the hip (a) sagittal, (b) frontal, (c) transverse planes; knee (d) sagittal, (e) frontal, and (f) transverse planes; ankle (g) sagittal and (h) transverse planes. SD, standard deviation. 
Thomas et al.

Table 2. Peak stance phase angles at the hip, knee, and ankle for pre- and post-fatigue states

\begin{tabular}{|c|c|c|c|c|c|c|c|c|}
\hline \multirow[t]{2}{*}{ Joint rotation $\left(^{\circ}\right)$} & \multicolumn{4}{|l|}{ Hip rotators } & \multicolumn{4}{|l|}{ Triceps surae } \\
\hline & Pre & Post & $P$ & $d$ & Pre & Post & $P$ & $d$ \\
\hline Hip flexion/extension & $44.6 \pm 6.3$ & $45.7 \pm 6.2$ & 0.42 & 0.17 & $47.0 \pm 7.4$ & $47.9 \pm 7.9$ & 0.16 & 0.13 \\
\hline Hip add/abduction & $-9.5 \pm 9.2$ & $-8.9 \pm 7.0$ & 0.42 & 0.07 & $-9.1 \pm 6.7$ & $-9.2 \pm 6.9$ & 0.89 & 0.01 \\
\hline Hip internal/external rotation & $9.8 \pm 7.5$ & $12.4 \pm 9.4$ & $0.04^{*}$ & 0.34 & $8.6 \pm 7.2$ & $9.2 \pm 8.7$ & 0.47 & 0.08 \\
\hline Knee extension/flexion & $-55.5 \pm 6.9$ & $-56.5 \pm 7.5$ & 0.42 & 0.15 & $-58.0 \pm 8.2$ & $-55.4 \pm 6.3$ & 0.06 & 0.31 \\
\hline Knee add/abduction & $-15.6 \pm 8.2$ & $-15.7 \pm 8.2$ & 0.91 & 0.01 & $-17.4 \pm 5.0$ & $-17.2 \pm 5.3$ & 0.70 & 0.04 \\
\hline Knee internal/external rotation & $4.6 \pm 7.9$ & $4.7 \pm 8.3$ & 0.92 & 0.01 & $2.1 \pm 5.4$ & $2.1 \pm 5.3$ & 0.97 & 0.00 \\
\hline Ankle dorsiflexion/plantar flexion & $14.5 \pm 5.7$ & $15.1 \pm 6.1$ & 0.57 & 0.10 & $14.5 \pm 6.1$ & $13.7 \pm 6.5$ & 0.48 & 0.12 \\
\hline Ankle inversion/eversion & $-8.8 \pm 7.5$ & $-9.5 \pm 7.0$ & 0.22 & 0.08 & $-8.7 \pm 5.7$ & $-8.7 \pm 5.2$ & 0.98 & 0.00 \\
\hline
\end{tabular}

All values are presented as mean \pm standard deviation.

The first rotation listed is positive (e.g., at peak the hip is internally rotated as indicated by the positive values).

${ }^{*}$ Statistically significant difference between fatigue conditions.

Table 3. Peak stance phase moments at the hip, knee, and ankle for pre- and post-fatigue states

\begin{tabular}{|c|c|c|c|c|c|c|c|c|}
\hline \multirow[t]{2}{*}{ Joint rotation $\left({ }^{\circ}\right)$} & \multicolumn{4}{|l|}{ Hip rotators } & \multicolumn{4}{|l|}{ Triceps surae } \\
\hline & Pre & Post & $P$ & $d$ & Pre & Post & $P$ & $d$ \\
\hline Hip flexion/extension & $0.88 \pm 0.59$ & $1.07 \pm 0.29$ & 0.21 & 0.32 & $1.01 \pm 0.36$ & $1.00 \pm 0.31$ & 0.69 & 0.03 \\
\hline Hip add/abduction & $-0.42 \pm 0.21$ & $-0.39 \pm 0.26$ & 0.61 & 0.14 & $-0.31 \pm 0.18$ & $-0.32 \pm 0.13$ & 0.68 & 0.06 \\
\hline Hip internal/external rotation & $0.38 \pm 0.10$ & $0.39 \pm 0.15$ & 0.64 & 0.10 & $0.37 \pm 0.12$ & $0.38 \pm 0.12$ & 0.26 & 0.08 \\
\hline Knee extension/flexion & $-1.67 \pm 0.38$ & $-1.57 \pm 0.31$ & 0.08 & 0.26 & $-1.66 \pm 0.35$ & $-1.64 \pm 0.25$ & 0.71 & 0.06 \\
\hline Knee add/abduction & $-0.82 \pm 0.29$ & $-0.77 \pm 0.23$ & 0.23 & 0.17 & $-0.69 \pm 0.48$ & $-0.75 \pm 0.20$ & 0.65 & 0.13 \\
\hline Knee internal/external rotation & $0.11 \pm 0.07$ & $0.11 \pm 0.06$ & 0.65 & 0.00 & $0.09 \pm 0.07$ & $0.08 \pm 0.05$ & 0.13 & 0.14 \\
\hline Ankle dorsiflexion/plantar flexion & $1.33 \pm 0.26$ & $1.25 \pm 0.22$ & 0.82 & 0.31 & $1.15 \pm 0.77$ & $1.25 \pm 0.20$ & 0.63 & 0.13 \\
\hline Ankle inversion/eversion & $-0.09 \pm 0.09$ & $-0.11 \pm 0.06$ & 0.43 & 0.22 & $-0.10 \pm 0.05$ & $-0.10 \pm 0.05$ & 0.59 & 0.00 \\
\hline
\end{tabular}

All values are presented as mean \pm standard deviation.

The first moment listed is positive (e.g., there is a hip flexion moment as indicated by the positive values).

Statistically significant difference between fatigue conditions.

disagreement with our hypothesis and this suggestion, knee abduction postures and loads did not concurrently increase. Previous research utilizing a general fatigue protocol indeed demonstrated concomitant increases in hip IR angle and knee abduction moment (Borotikar et al., 2008). In that study, however, multiple muscle groups that contribute to knee frontal plane loading control (e.g., quadriceps, hamstrings, gastrocnemius) were likely fatigued (Borotikar et al., 2008). With isolated hip rotator fatigue, therefore, pre-fatigue knee abduction biomechanics may have been maintained through the combined actions of these non-fatigued muscles. Integrated assessment of lower extremity muscle activation strategies during these tasks, both fatigued and non-fatigued, would provide additional insights here.

\section{Triceps surae fatigue}

Subjects landed with less IC knee flexion following triceps surae fatigue, likely representing a compensatory strategy to protect against lower limb collapse under loading. With the gastrocnemius primarily serving to eccentrically control ankle dorsiflexion during deceleration (Nyland et al., 1997), increased dorsiflexion following triceps surae fatigue would seem likely. We did not, however, observe this altered ankle response. Landing with less knee flexion may have assisted in preventing such an outcome, stiffening the lower limb to afford a more upright landing posture. This strategy may be problematic for ACL injury, however, as a less flexed knee upon landing may additionally precipitate extreme anterior tibial shear loads (Chappell et al., 2005). The gastrocnemius also assists the hamstrings in opposing anterior drawer of the tibia, driven largely by the extreme quadriceps loads associated with landings (Lass et al., 1991). The gastrocnemius, therefore, may afford a more quadriceps dominant landing strategy, potentially further increasing ACL loading and injury risk (DeMorat et al., 2004). Despite substantial triceps surae fatigue, subjects did not decrease postfatigue plantar flexion moment magnitudes. While in disagreement with our hypothesis, this may represent a critical performance-dependent response, wherein lower limb neuromuscular control shifted proximally following fatigue to ensure successful task execution. 
Hip and ankle fatigue alters kinematics

(a)

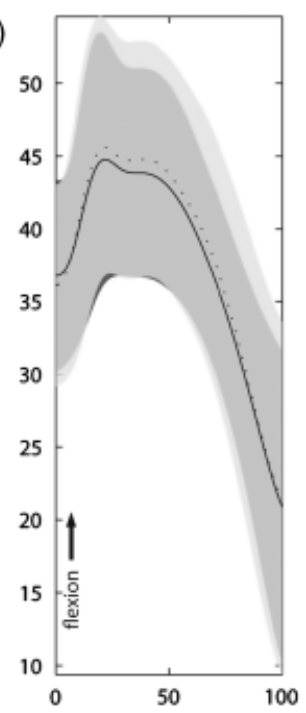

(d)

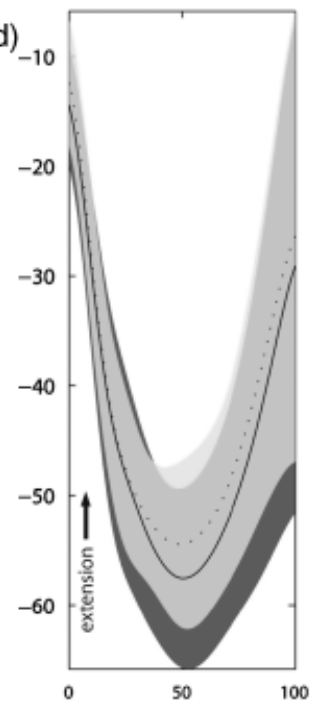

(b)

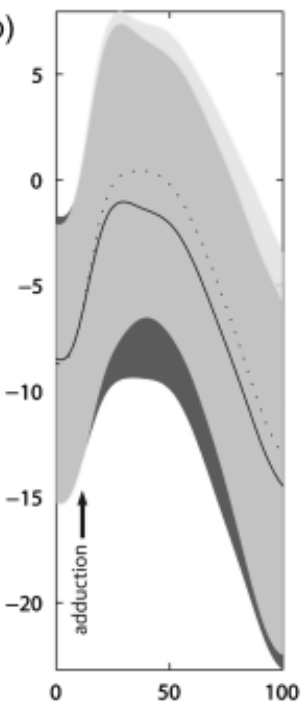

(e)

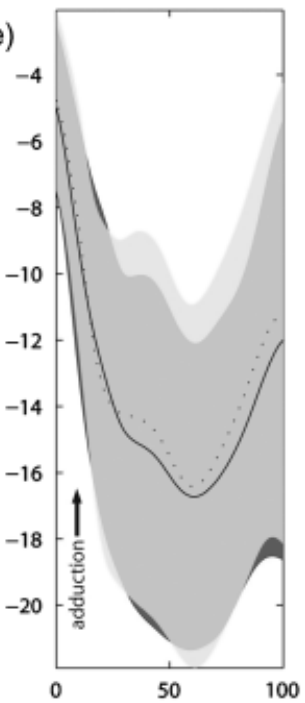

(c) 15

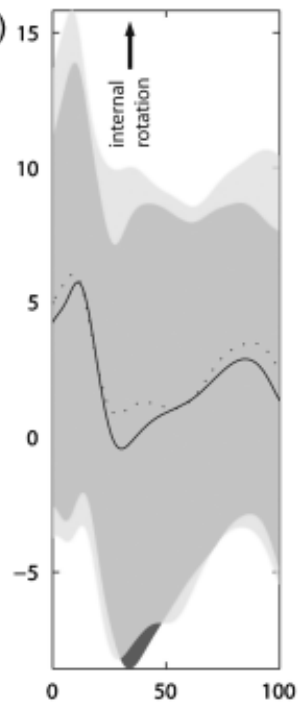

(f)

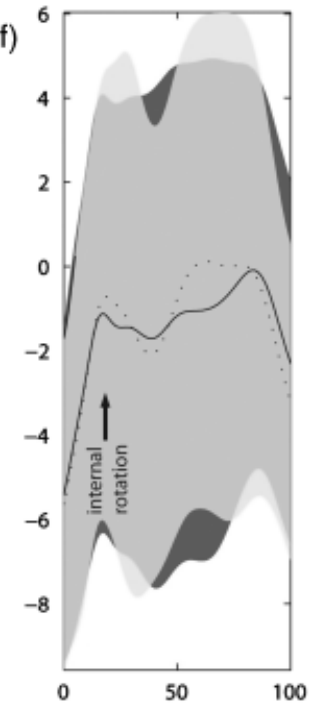

(g)

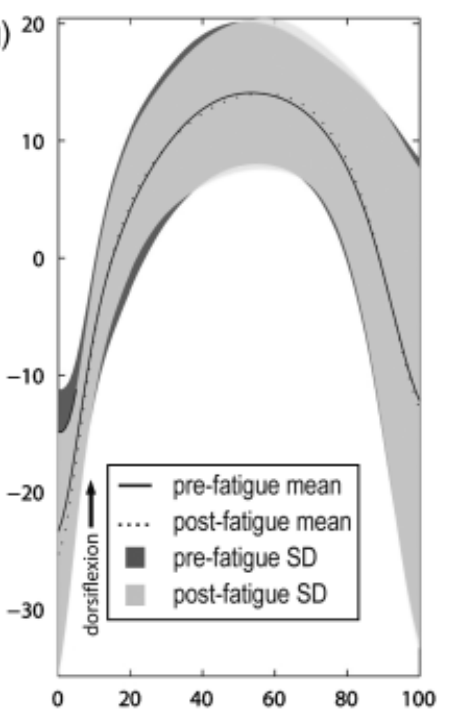

(h)

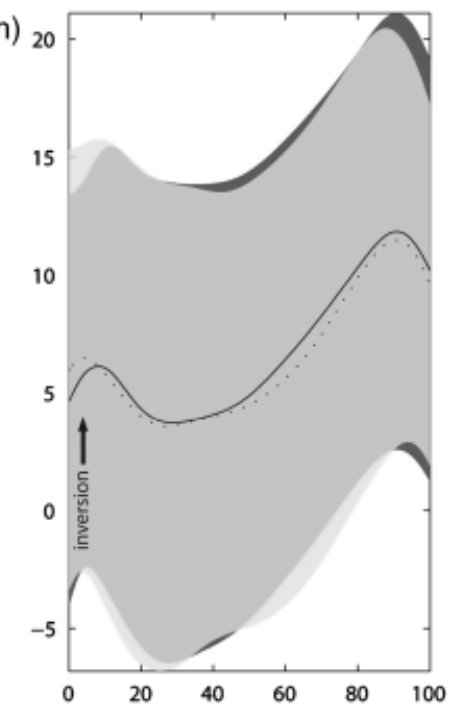

Fig. 3. Average joint rotations pre- and post-fatigue of the triceps surae. Rotations shown in the hip (a) sagittal, (b) frontal, and (c) transverse planes; knee (d) sagittal, (e) frontal, and (f) transverse planes; ankle (g) sagittal and (h) transverse planes. SD, standard deviation. 


\section{Thomas et al.}

As previously discussed, subjects demonstrated a decreased knee flexion angle at IC, allowing for a more upright posture on landing. We observed a similar trend towards this posture at PS, which may continue to afford an effective ankle strategy for task execution. As the landing task required performance of a lateral hop, necessitating push-off at the ankle, maintenance of a plantar flexion moment seems crucial.

Isolated fatigue of both the hip rotators and triceps surae-induced kinematic changes potentially associated with non-contact ACL injury. With ACL injury increasingly suggested to arise through combined 3D loading, however (Markolf et al., 1995; McLean et al., 2005), it seems unlikely that the uniplanar kinematic changes stemming from hip rotator or triceps surae fatigue represent risk in isolation. Additionally, the magnitude of these kinematic modifications was small and would likely not be sufficient to precipitate ACL injury. Rather, fatigue contributions to ACL injury more likely manifest via more gross lower extremity adaptations, wherein extreme 3D knee joint loading profiles are possible (McLean et al., 2007; McLean \& Samorezov, 2009). While assessments of isolated muscle fatigue may not elucidate injury mechanisms explicitly, it does afford assessment of the contributions of specific muscles to the overall neuromechanical profile. This knowledge is critical, for example, in determining the impact of weakness within a particular muscle group to that profile and whether targeted muscle strengthening may be warranted. Based on current results, however, it appears that since isolated muscle fatigue, and, hence, weakness does not signify extreme ACL injury risk, then targeted strengthening of individual muscle groups within the prevention modality may not be beneficial. Rather, muscles should be strengthened synergistically and trained dynamically in order to more effectively counter the complex 3D load states likely associated with ACL injury. Further, considering the intricate muscle activation strategies governing dynamic landing strategies (Besier et al., 2003), training aimed at coordinating muscle actions, rather than simply strengthening, may be more beneficial.

Specific kinetic and kinematic changes have been demonstrated previously to increase injury risk (Markolf et al., 1995; Hewett et al., 1996). While we based our consideration of injury risk on these previous studies, the precise magnitude of injurious biomechanics necessary to tear the ACL and, therefore the clinical meaningfulness of the relatively small kinematic changes $\left(2^{\circ}-3^{\circ}\right)$ in our study, is currently unknown. Additionally, inter-individual structural differences have been demonstrated to impact the resultant ligamentous loading during dynamic activity (Mizuno et al., 2009), making it difficult to ascertain the magnitude of biomechanical changes necessary to induce injury across individuals. Future research is clearly needed to elucidate the magnitude of biomechanical adaptations necessary to induce ACL injury and how sensitive this is to individual variations in structure and joint mechanics.

Several limitations exist with this study. We chose, for example, to induce fatigue through isolated concentric muscle contractions, primarily for consistency with our previous work (Thomas et al., in press). During the first half of landing, when rapid deceleration of the body segments occurs, the hip rotators and triceps surae musculature are likely contracting eccentrically to assist in dynamic stabilization. Therefore, fatiguing these muscle groups eccentrically could have yielded different results than did fatiguing them concentrically, as we have done presently. Extending assessments to include eccentric fatigue effects should thus be considered in future work in this area. Additionally, we chose to fatigue the musculature using an angular velocity of $180^{\circ} / \mathrm{s}$. While this velocity may not be reflective of that experienced during more dynamic, athletic activity, the impact of the angular velocity utilized on the resultant movement strategy is unknown and, thus we cannot be certain if the angular velocity chosen affected our outcomes. The knee joint angle utilized during the triceps surae fatiguing exercise may also be a limitation. We placed the knee in approximately $30^{\circ}$ of knee flexion, a position that may have resulted in greater fatigue within the soleus than the gastrocnemius. Finally, trunk position during landing tasks has been shown recently to directly influence lower limb biomechanics (Kulas et al., 2008). As such, it is plausible that for the post-fatigue condition, subjects altered their trunk position to perform the landing task successfully, which, in turn, may have confounded joint kinematic outcomes and resultant statistical comparisons. We did not currently quantify trunk position data and, thus, can merely speculate as to whether this variable influenced our results. An integrative assessment of trunk postures pre- and post-fatigue in our future work will alleviate this potential concern.

\section{Perspectives}

Isolated hip rotator and triceps surae fatigue produced specific hip and knee kinematic changes during landing. These adaptations in isolation, however, do not appear overly hazardous to the ACL. Rather, ACL injury appears more likely when exposed to gross lower extremity fatigue, where extreme modifications in the combined 3D knee loading state arise. Further research elucidating the precise role of neuromuscular fatigue within the non-contact ACL injury mechanism 
Hip and ankle fatigue alters kinematics

is needed so effective strategies to combat its hazardous effects can be developed and implemented.

Key words: biomechanics, ACL, fatigue.

\section{Acknowledgements}

The authors wish to thank Eric Boye, Kellen Lawless, and Ganapriya Venkatasubramanian, MS for assistance with data collection and processing.

\section{References}

Agel J, Arendt EA, Bershadsky B. Anterior cruciate ligament injury in national collegiate athletic association basketball and soccer: a 13-year review. Am J Sports Med 2005: 33: 524-530.

Andersson H, Raastad T, Nilsson J, Paulsen G, Garthe I, Kadi F. Neuromuscular fatigue and recovery in elite female soccer: effects of active recovery. Med Sci Sports Exerc 2008: 40: 372-380.

Bellew JW, Fenter PC. Control of balance differs after knee or ankle fatigue in older women. Arch Phys Med Rehabil 2006: 87: 1486-1489.

Besier TF, Lloyd DG, Ackland TR. Muscle activation strategies at the knee during running and cutting maneuvers. Med Sci Sports Exerc 2003: 35: 119127.

Besier TF, Lloyd DG, Cochrane JL, Ackland TR. External loading of the knee joint during running and cutting maneuvers. Med Sci Sports Exerc 2001: 33: 1168-1175.

Boden BP, Dean GS, Feagin JA Jr., Garrett WE Jr. Mechanisms of anterior cruciate ligament injury. Orthopedics 2000: 23: 573-578.

Borotikar BS, Newcomer R, Koppes R, McLean SG. Combined effects of fatigue and decision making on female lower limb landing postures: central and peripheral contributions to ACL injury risk. Clin Biomech (Bristol, Avon) 2008: 23: 81-92.

Bradley JP, Klimkiewicz JJ, Rytel MJ, Powell JW. Anterior cruciate ligament injuries in the National Football League: epidemiology and current treatment trends among team physicians. Arthroscopy 2002: 18: 502-509.

Chappell JD, Herman DC, Knight BS, Kirkendall DT, Garrett WE, Yu B. Effect of fatigue on knee kinetics and kinematics in stop-jump tasks. Am $\mathbf{J}$ Sports Med 2005: 33: 1022-1029.

Cohen J. Statistical power analysis for the behavioral sciences. New York: Academic Press, 1969: 415.

Cole GK, Nigg BM, Ronsky JL, Yeadon MR. Application of the joint coordinate system to three-dimensional joint attitude and movement representation: a standardization proposal. J Biomech Eng 1993: 115 : 344-349.
Cresswell AG, Loscher WN, Thorstensson A. Influence of gastrocnemius muscle length on triceps surae torque development and electromyographic activity in man. Exp Brain Res 1995: 105: 283-290.

Decker MJ, Torry MR, Wyland DJ, Sterett WI, Richard Steadman J. Gender differences in lower extremity kinematics, kinetics and energy absorption during landing. Clin Biomech (Bristol, Avon) 2003: 18: $662-$ 669.

Delp SL, Hess WE, Hungerford DS, Jones LC. Variation of rotation moment arms with hip flexion. J Biomech 1999: 32: 493-501.

DeMorat G, Weinhold P, Blackburn T, Chudik S, Garrett W. Aggressive quadriceps loading can induce noncontact anterior cruciate ligament injury. Am J Sports Med 2004: 32: $477-$ 483.

Dempster WT. Space requirements of seated operator. WADC Technical report, 1955: TR-55-59.

Ford KR, Myer GD, Hewett TE. Valgus knee motion during landing in high school female and male basketball players. Med Sci Sports Exerc 2003: 35: 1745-1750.

Ford KR, Myer GD, Smith RL, Vianello RM, Seiwert SL, Hewett TE. A comparison of dynamic coronal plane excursion between matched male and female athletes when performing single leg landings. Clin Biomech (Bristol, Avon) 2006: 21: 33-40.

Ford KR, Myer GD, Toms HE, Hewett TE. Gender differences in the kinematics of unanticipated cutting in young athletes. Med Sci Sports Exerc 2005: 37: 124-129.

Gandevia SC, Refshauge KM, Collins DF. Proprioception: peripheral inputs and perceptual interactions. Adv Exp Med Biol 2002: 508: 61-68.

Grood ES, Suntay WJ. A joint coordinate system for the clinical description of three-dimensional motions: application to the knee. J Biomech Eng 1983: 105: 136-144.

Hawkins RD, Fuller CW. A prospective epidemiological study of injuries in four English professional football clubs. Br J Sports Med 1999: 33: 196203.

Hewett TE, Lindenfeld TN, Riccobene JV, Noyes FR. The effect of neuromuscular training on the incidence of knee injury in female athletes. A prospective study. Am J Sports Med 1999: 27: 699-706.

Hewett TE, Myer GD, Ford KR. Anterior cruciate ligament injuries in female athletes: Part 1, mechanisms and risk factors. Am J Sports Med 2006: 34: 299-311.

Hewett TE, Stroupe AL, Nance TA, Noyes FR. Plyometric training in female athletes. Decreased impact forces and increased hamstring torques. Am J Sports Med 1996: 24: 765-773.

Ireland ML. The female ACL: why is it more prone to injury? Orthop Clin $\mathrm{N}$ Am 2002: 33: 637-651.

Kulas A, Zalewski P, Hortobagyi T, DeVita P. Effects of added trunk load and corresponding trunk position adaptations on lower extremity biomechanics during drop-landings. J Biomech 2008: 41: 180-185.

Lass P, Kaalund S, leFevre S, ArendtNielsen L, Sinkjaer T, Simonsen O. Muscle coordination following rupture of the anterior cruciate ligament. Electromyographic studies of 14 patients. Acta Orthop Scand 1991: 62: 9-14.

Markolf KL, Burchfield DM, Shapiro MM, Shepard MF, Finerman GA, Slauterbeck JL. Combined knee loading states that generate high anterior cruciate ligament forces. J Orthop Res 1995: 13: 930-935.

McLean SG, Felin R, Suedekum N, Calabrese G, Passerallo A, Joy S. Impact of fatigue on gender-based high-risk landing strategies.

Med Sci Sports Exerc 2007: 39: 502 514.

McLean SG, Huang X, van den Bogert AJ. Association between lower extremity posture at contact and peak knee valgus moment during sidestepping: implications for ACL injury. Clin Biomech 2005: 20: 863870.

McLean SG, Lipfert SW, van den Bogert AJ. Effect of gender and defensive opponent on the biomechanics of sidestep cutting. Med Sci Sports Exerc 2004: 36: 1008-1016.

McLean SG, Samorezov JE. Fatigueinduced ACL injury risk stems from a degradation in central control. Med Sci Sports Exerc 2009: 41: 1661-1672. 


\section{Thomas et al.}

Mizuno K, Andrish JT, van den Bogert AJ, McLean SG. Gender dimorphic ACL strain in response to combined dynamic 3D knee joint loading: implications for ACL injury risk. Knee 2009: 16: 432-440.

Nyland JA, Caborn DN, Shapiro R, Johnson DL. Fatigue after eccentric quadriceps femoris work produces earlier gastrocnemius and delayed quadriceps femoris activation during crossover cutting among normal athletic women. Knee Surg Sports Traumatol Arthrosc 1997: 5: 162-167.

Ochsendorf DT, Mattacola CG, Arnold BL. Effect of orthotics on postural sway after fatigue of the plantar flexors and dorsiflexors. J Athlet Train 2000: 35: 26-30.

Olsen OE, Myklebust G, Engebretsen L, Bahr R. Injury mechanisms for anterior cruciate ligament injuries in team handball: a systematic video analysis. Am J Sports Med 2004: 32: 1002-1012.

Thomas AC, McLean SG, Palmieri-Smith RM. Isolated quadriceps and hamstrings fatigue alters hip and knee mechanics. J Appl Biomech, in press.

Willson JD, Davis IS. Lower extremity mechanics of females with and without patellofemoral pain across activities with progressively greater task demands. Clin Biomech (Bristol, Avon) 2008: 23: 203-211.

Wu G, Siegler S, Allard P, Kirtley C, Leardini A, Rosenbaum D, et al. ISB recommendation on definitions of joint coordinate system of various joints for the reporting of human joint motion - part I: Ankle, hip, and spine. International Society of Biomechanics. J Biomech 2002: 35: 543-548.

Zeller BL, McCrory JL, Kibler WB, Uhl TL. Differences in kinematics and electromyographic activity between men and women during the singlelegged squat. Am J Sports Med 2003: 31: 449-456. 